\title{
Investigation on Nucleation Kinetics, Growth and Nonlinear Optical Properties of L-Cystine Hydrochloride Crystal
}

\author{
D. Manivannan ${ }^{a}$, K. Kirubavathi ${ }^{b}$ And K. Selvaraju ${ }^{b, *}$ \\ ${ }^{a}$ Department of Physics, Erode Arts and Science College (Autonomous), Erode - 638009, Tamilnadu, India \\ ${ }^{b}$ Post Graduate and Research Department of Physics, Government Arts College, Ariyalur-621713, Tamilnadu, India
}

(Received May 29, 2017; in final form November 13, 2017)

\begin{abstract}
The semiorganic nonlinear optical crystal of amino-carboxyl acid family, L-cystine hydrochloride (LCHCl) was successfully grown from its aqueous solution by the slow evaporation solution growth method. The solubility, metastable zone width and induction period were determined for the first time experimentally and there by the possibility of growing bulk crystals of $\mathrm{LCHCl}$ using deionized water as solvent. The induction period was recorded for the different supersaturation ratios $(S=1.2,1.3,1.4$, and 1.5$)$, which reveals that the induction period of $\mathrm{LCHCl}$ decreases with increase in supersaturation. The nucleation kinetic parameters such as critical free energy, change of volume free energy, critical radius, number of molecules in the critical nucleus and nucleation rate have been evaluated for $\mathrm{LCHCl}$ crystals. The interfacial energy values of $\mathrm{LCHCl}$ were determined for different supersaturation ratio by means of varying temperatures. The single crystal X-ray diffraction gives the lattice parameters value of the grown crystals. The second harmonic generation efficiency was confirmed by the Kurtz-Perry powder method. The laser damage threshold energy of the grown crystal indicates that grown crystal has excellent resistance to laser radiation also compared with known other nonlinear optical crystals.
\end{abstract}

DOI: 10.12693/APhysPolA.133.63

PACS/topics: crystal growth, nucleation kinetics, optical materials, NLO

\section{Introduction}

In the recent years, second order nonlinear optical (NLO) materials were found capable of competent frequency conversion to blue-violet and ultraviolet wavelengths by means of laser diodes. These materials are found in various applications particularly in photonics technology, optical data storage, and laser spectroscopy [1,2]. Also having high second order optical nonlinearities, wide transparency window with low range cutoff wavelength, with chemical, mechanical and thermal stability are totally necessary, apart from the other requirements, in order to be conscious of these applications [3].

L-cystine hydrochloride $(\mathrm{LCHCl})$ is a recently discovered second order nonlinear optical material. The second harmonic generation efficiency of $\mathrm{LCHCl}$ crystal was 1.2 times that of potassium hydrogen phosphate (KDP) [4]. The crystal structure of L-cystine hydrochloride has been reported previously [5-7]. It belongs to the monoclinic crystallographic system, space group $C 2$, with cell parameters: $a=18.63, b=5.26, c=7.28 \AA, \beta=103.7$, $Z=2$. Although, the growth aspects of $\mathrm{LCHCl}$ crystal have been reported in our laboratory [4] and few reports are presented on $\mathrm{LCHCl}$ based NLO crystals [8-11]. In order to grow crystals with optical qualities for nonlinear and device applications, a systematic investigation of optimized growth conditions has been carried out. The fundamental nucleation parameters are requisite for the

*corresponding author; e-mail: selsphy@yahoo.com growth of crystals by the solution growth technique. The homogeneous nucleation theory [12-17] is used to calculate the nucleation parameters from aqueous solutions. In the literature, there is no report of nucleation kinetics and laser damage threshold analysis of this $\mathrm{LCHCl}$ crystal. Due to this, we are very much concerned a systematic investigation of optimized growth conditions of the crystals.

In the present investigations, attempts have been made to examine the nucleation parameters of $\mathrm{LCHCl}$, which are the prerequisite for investigating the growth mechanism of bulk size single crystals. The solubility, metastable zone width and interfacial surface tension of $\mathrm{LCHCl}$ have been effectively determined for the first time. The induction period was also measured for various supersaturation ratios by a constant temperature. The X-ray diffraction (XRD) and laser damage threshold studies are also studied.

\section{Experimental studies}

2.1. Solubility studies of $\mathrm{LCHCl}$

The salt of $\mathrm{LCHCl}$ was synthesized by using AR grade chemicals as per the stoichiometric ratio. The growth rate of a crystal depends on its solubility and its corresponding temperature. The solubility of $\mathrm{LCHCl}$ was carried out in a solvent of deionized water at five different temperatures $\left(35,40,45,50\right.$ and $\left.55^{\circ} \mathrm{C}\right)$. The solubility studies were examined by dissolving the synthesized salt of $\mathrm{LCHCl}$ in $100 \mathrm{ml}$ of mixture solvent at a constant temperature with constant stirring. Later than attaining the saturation, the equilibrium concentration of the solute has been analyzed through gravimetrically. The similar process was repeated at different temperatures. Figure 1 shows the solubility graph of $\mathrm{LCHCl}$ crystal. From Fig. 1, 
it is observed that the solubility significantly increases with increase of the temperature, which gives the high positive solubility and temperature gradient suggesting that these crystals are suitable for the growth of slow cooling as well as the slow evaporation method.

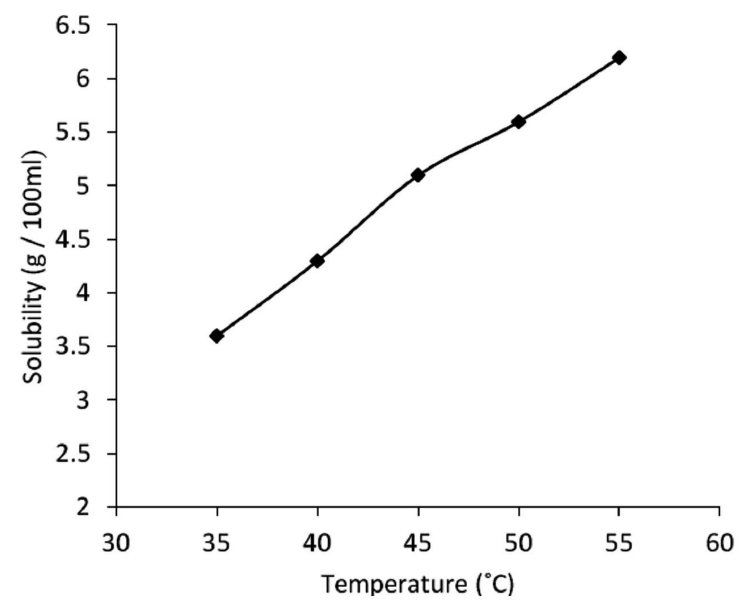

Fig. 1. Solubility curve of $\mathrm{LCHCl}$ crystal.

\subsection{Metastable and induction period measurements}

From the crystal growth point of view, the stability of the solution is associated with the metastable zone width also the higher zone width suggests that the stability of the solution will be greater [18]. Metastable zone width or Ostwald-Meirs region is defined as the degree of supercooling $\left(\Delta T_{c}\right)$ needed for one nucleus to be formed per unit volume in one second or the critical supercooling $\Delta T_{c}$ required to grow one nucleus per unit volume is called metastable zone width. To determine metastable zone width, the saturated solution of L-cystine hydrochloride has been prepared from the solubility studies. The study was carried out at a constant temperature bath with an accuracy of $\pm 0.01^{\circ} \mathrm{C}$ provided with a cryostat for cooling below the room temperature. A constant volume of $100 \mathrm{ml}$ of the solution was used in all the measurements. The solution was prepared $5{ }^{\circ} \mathrm{C}$ above the saturation temperature for homogenization and left out at the superheated temperature for $1 \mathrm{~h}$ before cooling it $[19,20]$. The solution was continuously stirred using an immersible stirrer to make the homogeneous concentration and temperature throughout the entire volume of the solution.

The metastable zone width of $\mathrm{LCHCl}$ was measured using the conventional polythermal method [21, 22]. In this method, the equilibrium-saturated solution was cooled from the overheated temperature until the first visible crystal was identified. Since the time taken for the development of the first visible crystal after the achievement of this, may be taken as the critical nucleus. The metastable zone width of $\mathrm{LCHCl}$ as a function of temperature is shown in Fig. 2.

Induction period $\tau$ of crystallization is generally defined as the time taken from the attainment of supersat-

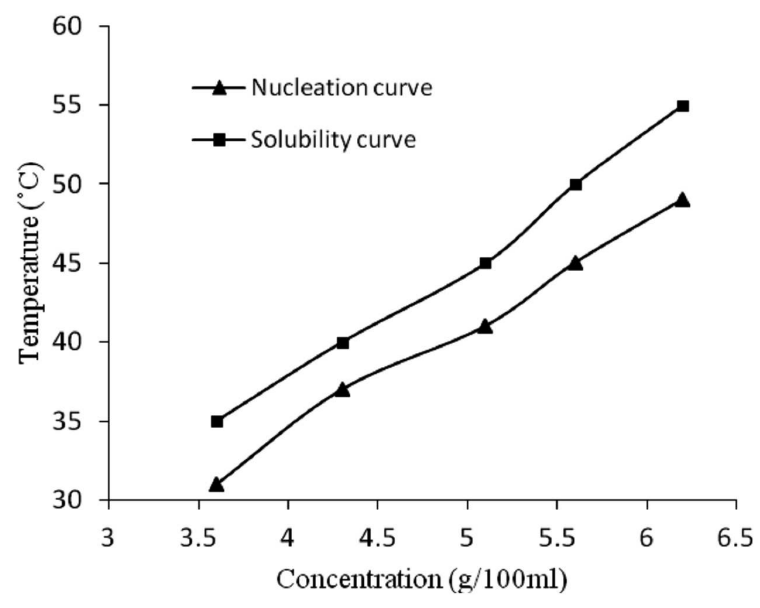

Fig. 2. Metastable zone width of $\mathrm{LCHCl}$ as a function of temperature.

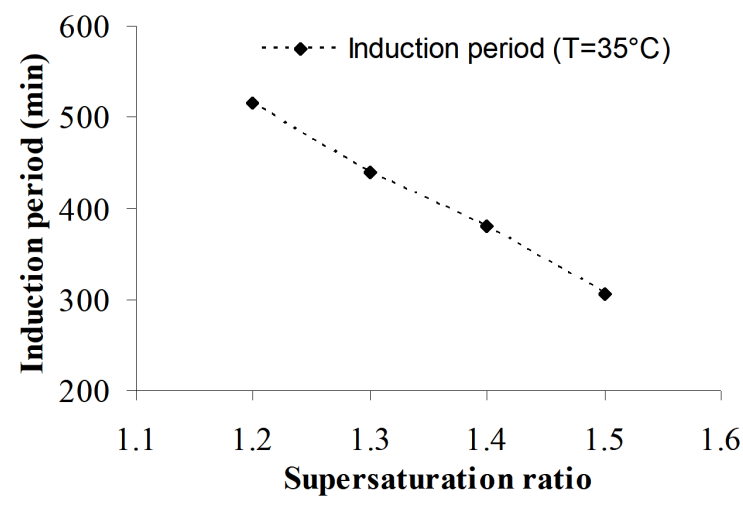

Fig. 3. Induction period versus supersaturation ratio of $\mathrm{LCHCl}$.

uration and the detection of critical nucleus [23]. Therefore, measurement of induction time requires determination of two points in time, which is the time interval between the achievement of supersaturation and the appearance of a crystal in noticeable size. The induction period versus supersaturation ratio of $\mathrm{LCHCl}$ is shown in Fig. 3.

\subsection{Nucleation kinetics of $\mathrm{LCHCl}$ crystal}

The crystallization stimulates because the creation of driving force due to the change in the Gibbs free energy $(\Delta G)$ between the crystalline phase with surrounding mother liquor. For rapid crystallization, $\Delta G<0$, the energy required to form $\mathrm{LCHCl}$ nucleus is given by

$$
\Delta G=\frac{4}{3} \pi r^{3} \Delta G v+4 \pi r^{2} \sigma,
$$

where $\Delta G_{v}$ is the energy per unit volume, $\sigma$ is the interfacial energy and $r$ is the radius of the nucleus. In the above equation the first term gives the formation of the new surface and the second term expresses the difference in the chemical potential between the crystalline phase and the surrounding mother liquor. At critical state, the free energy formation obeys the condition $\frac{\mathrm{d}(\Delta G)}{\mathrm{d} r}=0$.

As per the regular solution theory, Bennema and 
Sohnel [24] have derived the expression for the linear dependence of in solubility

$$
\sigma=\frac{k T}{d^{2}}\left(0.173-0.248 \ln \left(x_{m}\right)\right),
$$

where $x_{m}$ is the mole fraction of the solute, $T$ is the temperature in $\mathrm{K}, d$ is the interionic distance and $k$ is the Boltzmann constant $1.38 \times 10^{-23} \mathrm{~J} / \mathrm{K}$.

The $\mathrm{LCHCl}$ crystal interfacial energy value has been found at various temperatures from the solubility data. The calculated range of interfacial energy is 1.1165 to $1.9958 \mathrm{~mJ} / \mathrm{m}^{2}$.

The radius of the critical nucleus is derived from

$$
r^{*}=-\left[\frac{2 \sigma}{\Delta G v}\right] \text {. }
$$

The driving force for the nucleation from the supersaturated solution is

$$
\Delta G v=-\left(\frac{k T}{V}\right) \ln S ; S=C / C^{*} .
$$

The critical energy barrier associated with the critical nucleation is related through

$$
\Delta G^{*}=\frac{4}{3} \pi r^{2} .
$$

The number of molecules in the critical nucleus is expressed as

$$
i^{*}=\frac{4 \pi r^{* 3}}{3 V} .
$$

The nucleation rate $J$ has been calculated using the equation

$$
J=A \exp \left(\frac{-\Delta G^{*}}{k T}\right),
$$

where $A$ is the pre-exponential factor $\left(\approx 10^{30}\right.$ for solution).

Based on the above formalism, the critical radius $\left(r^{*}\right)$, critical free energy $\left(\Delta G^{*}\right)$, volume free energy $\left(\Delta G_{v}\right)$, number of molecules in the critical nucleus $\left(i^{*}\right)$ and nucleation rate $(J)$ have been calculated at various supersaturation values and are given in Table I.

TABLE I

Nucleation parameters of LCHCl.

\begin{tabular}{c|c|c|c|c|c}
\hline \hline$S$ & $\begin{array}{c}\Delta G_{v} \\
{\left[\frac{\mathrm{Mg}}{\mathrm{cm}^{3}}\right]}\end{array}$ & $\begin{array}{c}r^{*} \\
{\left[10^{-10} \mathrm{~m}\right]}\end{array}$ & $\begin{array}{c}\Delta G^{*} \\
{\left[10^{-20} \frac{\mathrm{mJ}}{\mathrm{m}^{3}}\right]}\end{array}$ & $i^{*}$ & $\begin{array}{c}J \\
{\left[\frac{\mathrm{n}}{\mathrm{svol}}\right]}\end{array}$ \\
\hline 1.2 & -3.6890 & 6.0535 & 17.1304 & 30.7203 & $6.6829 \times 10^{21}$ \\
1.3 & -4.7310 & 5.1338 & 13.3675 & 18.7398 & $7.3015 \times 10^{21}$ \\
1.4 & -5.7010 & 4.5798 & 11.4644 & 13.3042 & $5.635 \times 10^{24}$ \\
1.5 & -6.6085 & 4.2088 & 10.3139 & 10.3258 & $9.3453 \times 10^{30}$
\end{tabular}

The nucleation kinetics is calculated and presented in this study give a deep approach to the nucleation process of low temperature solution growth of L-cystine hydrochloride crystal. Investigations carried out using homogeneous nucleation theory provide a clear picture on the permanence of the solution to the aquo solvent. Interfacial energy of a solution is directly related to the solubility and it is also inversely proportional to the growth rate of the crystal. Therefore, it is concluded that the interfacial energy plays a major role in the nucleation process.

The induction period with respect to supersaturation ratio is shown in Fig. 3. From the figure, it is understanding that the induction period decreases from higher supersaturation ratios. It gives the number of critical nuclei formation will increase, to lead the spurious nucleation. The study of induction period against supersaturation ratio gives an idea of optimized induction period in order to have controlled nucleation rate to grow good optical quality single crystals.

\subsection{Crystal growth}

From the solubility data, the calculated amounts of L-cystine and hydrochloric acid were systematically dissolved in double distilled water and simultaneous heating at room temperature. The vigorous stirring was continuous for $4-5 \mathrm{~h}$ to get a saturated solution. The prepared saturated solution was purified by filtering through the fine pores using Whatman filter paper. The purity of synthesized materials of $\mathrm{LCHCl}$ was increased using the recrystallization method. The homogeneous prepared saturated solution was filtered and transferred to new cleaned crystal growth vessel and closed with perforated sheets to evaporate slowly. Using the slow evaporation technique, well developed optical quality single crystal of $\mathrm{LCHCl}$ was obtained within 30 days, as grown crystal shown in Fig. 4.

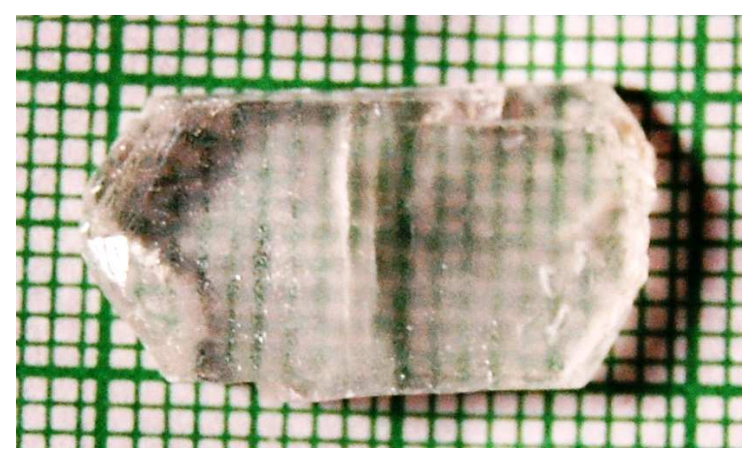

Fig. 4. Transparent LCHCl crystal grown under optimized nucleation parameters.

\section{Single crystal XRD}

The lattice parameters and the crystal systems have been determined using single crystal X-ray diffraction analysis. The single crystal X-ray diffraction analysis for the grown $\mathrm{LCHCl}$ crystals has been carried out using an ENRAF NONIUS CAD 4 automatic X-ray diffractometer. Single crystal XRD data confirms that the crystal belongs to monoclinic crystal system. The calculated lattice parameters are: $a=18.656, b=5.385, c=7.612$ Aand $\beta=103.40$ which agree well with the previously reported values [5-7]. Also it is reported [5-7] that the space group $C_{2}$ is recognized as a non-centrosymmetric and thus, satisfying one of the basic and essential material requirements for the second harmonic generation activity of the crystal. 


\section{NLO}

The powder second harmonic generation (SHG) efficiency of LCHCl crystal was examined using the Kurtz and Perry [25] powder technique. The schematic diagram of Kurtz and Perry powder SHG measurement is shown in Fig. 5. The powdered sample of $\mathrm{LCHCl}$ is illuminated by the fundamental beam of Nd:YAG laser $(1064 \mathrm{~nm})$. The input pulse width was $8 \mathrm{~ns}$ and $0.6 \mathrm{~J} /$ pulse energy was used with a repetition rate of $10 \mathrm{~Hz}$. The second harmonic signal generated was confirmed from the green light emission of wavelength $532 \mathrm{~nm}$ from the powdered $\mathrm{LCHCl}$ sample. Using the monochromator the $532 \mathrm{~nm}$ radiation was collected after separating from the $1064 \mathrm{~nm}$ input beam with IR filter. The SHG output $(532 \mathrm{~nm})$ is finally detected by a photomultiplier tube which was converted into voltage output displayed on the oscilloscope. Similarly, the potassium dihydrogen phosphate (KDP) was also powdered nearly the same particle size of the experimental sample to use as a reference material in the present measurement. The output power of $\mathrm{LCHCl}$ was measured to be $15 \mathrm{mV}$. For the same input (Nd:YAG laser - $1064 \mathrm{~nm}$, pulse width $8 \mathrm{~ns}$ and $0.6 \mathrm{~J} /$ pulse energy), KDP emitted the green light with the output power of $12 \mathrm{mV}$. The SHG efficiency was found to be 1.2 times that of KDP.

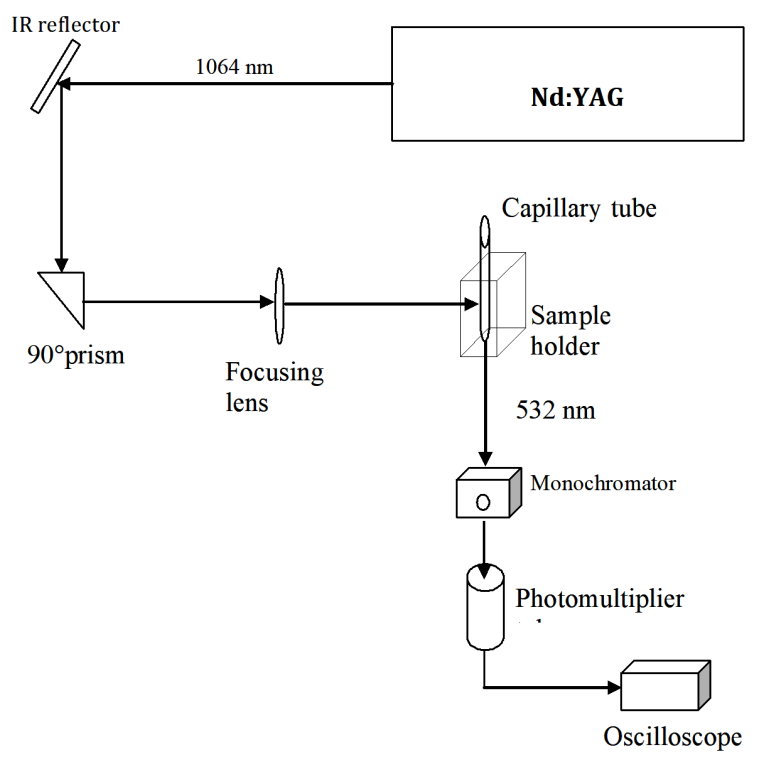

Fig. 5. Experimental setup for SHG measurement.

The main contribution to the quadratic NLO response from KDP crystal is made by the group of $\mathrm{PO}_{4}$ and the presence of hydrogen bonds. Also it has been shown that the NLO behaviour of KDP can be controlled due to increase in the number of hydrogen bonds between the crystal and their optimization of its spatial arrangement [26-29]. The delocalized electronic systems of aromatic organic and semiorganic molecules can result in much higher second-order nonlinear optical efficiencies than those of inorganic materials. Also which possess high polarizability and it contains the groups which can effectively form hydrogen bonds with the growing crystal face [30-32]. The SHG efficiency of LCHCl crystal depends upon the contribution to nonlinear polarizability of L-cystine molecules due to interamolecular charge transfer, the mobility of charge carriers through pi-bonding network and though presence of hydrogen bonds between L-cystine and hydrochloric acid. Also it has no extensive systems of pi-bonds, they possess intermolecular charge transfer of donor and acceptor groups. In view of this, it is clear that the $\mathrm{LCHCl}$ crystal has leads to improve (due to altering molecular arrangement) the SHG efficiency compared with KDP.

The comparative analysis of the SHG efficiency of other NLO crystals as L-valine hydrochloride has 1.7 times that of KDP (Nd:YAG laser $1064 \mathrm{~nm}$, pulse energy $4 \mathrm{~mJ} /$ pulse and pulse width of $10 \mathrm{~ns}$ and repetition rate of $10 \mathrm{~Hz}$ were used) [33]. L-histidine hydrochloride monohydrate has 3 times higher than that of KDP (input pulsed laser beam of Nd:YAG laser with a pulse width of $8 \mathrm{~ns}$ ) [34]. L-ornithine monohydrochloride powder SHG efficiency has 1.25 times that of KDP using Nd:YAG laser $(1064 \mathrm{~nm})$ with a pulse width of $8 \mathrm{~ns}$ and pulse of energy $4 \mathrm{~mJ}$ [35]. Also the inorganic crystal SHG efficiency is compared as $\mathrm{Cd}_{0.70} \mathrm{Mg}_{0.30}$ Se are about ten times larger than $\mathrm{Zn}_{0.67} \mathrm{Mg}_{0.33}$ Se crystal using Nd:YAG laser (Quantel 60 Model YG472) working at $532 \mathrm{~nm}$ with $30 \mathrm{ps}$ pulses duration and $1 \mathrm{~Hz}$ repetition rate [36].

\section{LDT}

Surface laser damage threshold (LDT) studies on NLO crystals are much important as the surface damage to these crystals by high power laser limits its performance in NLO applications. In the present study, the LDT value of LCHCl has been calculated by using a Q-switched pulsed Nd:YAG (1064 nm, $420 \mathrm{~mJ}, 10 \mathrm{~Hz}$ ) laser as a source. Using the variable attenuator the energy of the laser beam was controlled and focused on the focal length of $30 \mathrm{~cm}$. It was focused on the mounted crystal at the focal point of the lens. For this measurement, the diameter of the laser beam $1 \mathrm{~mm}$ at focal length allowed to irradiate the polished surface of the $\mathrm{LCHCl}$ crystal. The input energy density of the laser beam was measured at which the crystal gets damaged with a clear visible spot on the surface and audible sound using a power meter. The surface damage threshold of $\mathrm{LCHCl}$ crystal was calculated using the expression: power density $=E / A\left[\mathrm{GW} / \mathrm{cm}^{2}\right]$ where $E$ is the input energy $[\mathrm{mJ}]$ and $A$ is the area of the circular spot size [mm]. The measured surface laser damage threshold of $\mathrm{LCHCl}$ is $0.32 \mathrm{GW} / \mathrm{cm}^{2}$. The laser damage threshold value of $\mathrm{LCHCl}$ crystal was compared with some well-known NLO crystals and is given in Table II [37].

\section{Conclusions}

The single crystals of L-cystine hydrochloride were grown by slow evaporation method. The present investigations, the solubility, metastable zone width and induction period were calculated experimentally for the crys- 
TABLE II

Comparison of laser damage threshold value of $\mathrm{LCHCl}$ crystal with some well-known NLO crystals.

\begin{tabular}{c|c|c}
\hline \hline Crystal & LDT value $\left[\frac{\mathrm{GW}}{\mathrm{cm}^{2}}\right]$ & Pulse width $[\mathrm{ns}]$ \\
\hline $\mathrm{KDP}$ & 5.00 & 1 \\
$\mathrm{LiNbO}_{3}$ & 0.30 & 10 \\
$\mathrm{KNbO}_{3}$ & 1.00 & 10 \\
$\mathrm{LCHCl}$ & 0.32 & 10
\end{tabular}

tallization of $\mathrm{LCHCl}$ crystal. It was found that the induction period decreases from the increase in solution supersaturations. Also the nucleation kinetics and fundamental growth parameters such as critical radius, critical free energy, volume free energy change, number of molecules in the critical nucleus and nucleation rate are estimated. The experimental results show that the critical radius of nucleus and the critical energy barrier will decrease with increase of supersaturation. Based on these nucleation kinetics data, transparent bulk single crystal of $\mathrm{LCHCl}$ was successfully grown. The single crystal XRD revealed the respective cell parameters. The powder SHG test was confirmed using the Kurtz-Perry method, the observed SHG efficiency is 1.2 times than that of KDP. The laser damage threshold value of $\mathrm{LCHCl}$ crystal was found to be $0.32 \mathrm{GW} / \mathrm{cm}^{2}$ and this value was compared with some well-known NLO crystals.

\section{References}

[1] P.N. Prasad, D.J. Williams, Introduction to Nonlinear Optical Effects in Organic Molecules and Polymers, Wiley, New York 1991.

[2] C. Chen, N. Ye, L. Lin, J. Jiang, W. Zeng, B. Wu, Adv Mater. 11, 1071 (1999).

[3] S. Manivannan, S. Dhanuskodi, J. Cryst. Growth 262, 473 (2004).

[4] K. Selvaraju, R. Valluvan, K. Kirubavathi, S. Kumararaman, Opt. Commun. 269, 230 (2007).

[5] R. Srinivasan, Acta Crystallogr. 9, 1039 (1956).

[6] L.K. Steinrauf, L.H. Jensen, Acta Crystallogr. 9, 539 (1956).

[7] L.K. Steinrauf, J. Peterso, L.H. Jensen, J. Am. Chem. Soc. 80, 3835 (1958).

[8] T. Uma Devi, N. Lawrence, R. Ramesh Babu, S. Selvanayagam, H. Stoeckli-Evans, G. Bhagavannarayan, K. Ramamurthi, J. Min. Mater. Char. Eng. 9, 495 (2010).

[9] A.V. Mohammed, J.A. Pragasam Arulappan, T.G. Sunitha, J. Chem. Pharm. Res. 7, 838 (2015).

[10] R. Vasanthakumari, W. Nirmala, R. Santhakumari, Adv. Appl. Sci. Res. 6, 159 (2015).

[11] S.K. Chandran, R. Paulraj, P. Ramasamy, Spectrochim. Acta A 151, 432 (2015).

[12] A.E. Nilson, O.J. Sohnel, J. Cryst. Growth 11, 233 (1971).

[13] K. Sangwal, J. Cryst. Growth 97, 393 (1989).
[14] J. Scholl, L. Vicum, M. Muller, M. Mazzotti, Chem. Eng. Technol 29, 257 (2006).

[15] A. Mersmann, J. Cryst. Growth 102, 841 (1960).

[16] T. Raghavalu, G. Ramesh Kumar, S. Gokul Raj, V. Mathivanan, R. Mohan, J. Cryst. Growth 307, 112 (2007).

[17] K. Selvaraju, K. Kirubavathi, N. Vijayan, S. Kumararaman, J. Cryst. Growth 310, 2859 (2008).

[18] H.E. Buckley, Crystal Growth, Wiley, New York 1951.

[19] R. Mohan Kumar, N. Gopalakrishnan, R. Jayavel, P. Ramasamy, Cryst. Res. Technol. 34, 1265 (1999).

[20] P.M. Ushasree, R. Muralidharan, R. Jayavel, P. Ramasamy, J. Cryst. Growth 210, 741 (2000).

[21] J. Nyvlt, R. Rychly, J. Gottfried, J. Wurzelova, J. Cryst. Growth 6, 151 (1970).

[22] N.P. Zaitseva, L.N. Rashkovich, S.V. Bogatyatrva, J. Cryst. Growth 148, 276 (1995).

[23] O. Sohnel, J. Cryst. Growth 57, 101 (1982).

[24] P. Bennema, O. Sohnel, J. Cryst. Growth 102, 547 (1990).

[25] S.K. Kurtz, T.T. Perry, J. Appl. Phys. 39, 3798 (1968).

[26] Dongfeng Xue, Siyuan Zhang, Chem. Phys. Lett. 301, 449 (1999).

[27] Dongfeng Xue, Siyuan Zhang, Physica B 262, 78 (1999).

[28] I. Pritula, A. Kosinova, M. Kolybayeva, V. Puzikov, S. Bondarenko, V. Tkachenko, V. Tsurikov, O. Fesenko, Mater. Res. Bull. 43, 2778 (2008).

[29] Zhe-shuai Lin, Zhi-zhong Wang, Chung-tian Chen, Ming-Hsien Lee, J. Chem. Phys. 118, 2349 (2003).

[30] Ke-chen Wu, Ca-iping Liu, Chao-yong Mang, Opt. Mater. 29, 1129 (2007).

[31] I. Pritula, V. Gayvoronsky, Yu. Gromov, M. Kopylovsky, M. Kolybaeva, V. Puzikov, A. Kosinova, Yu. Savvin, Yu. Velikhov, A. Levchenko, Opt. Commun. 282, 1141 (2009).

[32] I.M. Pritula, Yu.N. Velikhov, Proc. SPIE 3793, 202 (1999).

[33] K. Kirubavathi, K. Selvaraju, R. Valluvan, N. Vijayan, S. Kumararaman, Spectrochim. Acta A 69, 1283 (2008)

[34] P. Anandan, R. Jayavel, T. Saravanan, G. Parthipan, C. Vedhi, R. Mohan Kumar, Opt. Mater. 34, 1225 (2012).

[35] T. Balakrishnan, K. Ramamurthi, Spectrochim. Acta A 72, 269 (2009).

[36] B. Derkowska, Z. Essaïdi, B. Sahraoui, A. Marasek, F. Firszt, M. Kujawa, Opt. Mater. 31, 518 (2009).

[37] H.L. Bhat, Bull. Mater. Sci. 17, 1233 (1994). 